\title{
A Giant Ly $\alpha$ Nebula and a Small-scale Clumpy Outflow in the System of the Exotic Quasar J0952+0114 Unveiled by MUSE*
}

\author{
Raffaella Anna Marino ${ }^{1}$ (D), Sebastiano Cantalupo ${ }^{1}$, Gabriele Pezzulli ${ }^{1}$, Simon J. Lilly ${ }^{1}$ (D), Sofia Gallego ${ }^{1}$, Ruari Mackenzie ${ }^{1}$, \\ Jorryt Matthee $^{1}$ (i), Jarle Brinchmann ${ }^{2,3}$ (i) , Nicolas Bouché ${ }^{4,5}$, Anna Feltre ${ }^{5,6}$, Sowgat Muzahid ${ }^{3}$ (D), Ilane Schroetter ${ }^{7}$ (D), \\ Sean D. Johnson ${ }^{8,9}$, and Themiya Nanayakkara ${ }^{3}$ (D) \\ ${ }^{1}$ Department of Physics, ETH Zürich, Wolfgang-Pauli-Strasse27, 8093Zürich, Switzerland; marinor@phys.ethz.ch \\ ${ }^{2}$ Instituto de Astrofísica e Ciências do Espaço, Universidade do Porto, CAUP, Rua das Estrelas, PT4150-762 Porto, Portugal \\ ${ }^{3}$ Leiden Observatory, Leiden University, P.O. Box 9513, NL-2300 RA, Leiden, The Netherlands \\ ${ }_{5}^{4}$ Institut de Recherche en Astrophysique et Planétologie (IRAP), Université de Toulouse, CNRS, UPS, F-31400 Toulouse, France \\ ${ }^{5}$ Univ. Lyon1, Ens de Lyon, CNRS, Centre de Recherche Astrophysique de Lyon UMR5574, F-69230 Saint-Genis-Laval, France \\ ${ }_{7}^{6}$ Scuola Internazionale Superiore di Studi Avanzati (SISSA), Via Bonomea 265, I-34136, Trieste, Italy \\ ${ }^{7}$ GEPI, Observatoire de Paris, PSL Université, CNRS, 5 Place Jules Janssen, F-92190 Meudon, France \\ ${ }^{8}$ Department of Astrophysical Science, 4 Ivy Lane, Princeton University, Princeton, NJ 08644, USA \\ ${ }^{9}$ The Observatories of the Carnegie Institution for Science, 813 Santa Barbara Street, Pasadena, CA 91101, USA \\ Received 2019 April 2; revised 2019 June 5; accepted 2019 June 8; published 2019 July 24
}

\begin{abstract}
The well-known quasar SDSS J095253.83+011421.9 (J0952+0114) at $z=3.02$ has one of the most peculiar spectra discovered so far, showing the presence of narrow Ly $\alpha$ and broad metal emission lines. Although recent studies have suggested that a proximate damped Ly $\alpha$ absorption (PDLA) system causes this peculiar spectrum, the origin of the gas associated with the PDLA is unknown. Here we report the results of observations with the Multi Unit Spectroscopic Explorer (MUSE) that reveal a new giant $(\approx 100$ physical kpc) Ly $\alpha$ nebula. The detailed analysis of the Ly $\alpha$ velocity, velocity dispersion, and surface brightness profiles suggests that the J0952+0114 Ly $\alpha$ nebula shares similar properties with other QSO nebulae previously detected with MUSE, implying that the PDLA in J0952+0144 is covering only a small fraction of the solid angle of the QSO emission. We also detected bright and spectrally narrow C IV $\lambda 1550$ and He II $\lambda 1640$ extended emission around J0952+0114 with velocity centroids similar to the peak of the extended and central narrow Ly $\alpha$ emission. The presence of a peculiarly bright, unresolved, and relatively broad He II $\lambda 1640$ emission in the central region at exactly the same PDLA redshift hints at the possibility that the PDLA originates in a clumpy outflow with a bulk velocity of about $500 \mathrm{~km} \mathrm{~s}^{-1}$. The smaller velocity dispersion of the large-scale Ly $\alpha$ emission suggests that the high-speed outflow is confined to the central region. Lastly, the derived spatially resolved He II/Ly $\alpha$ and C IV/Ly $\alpha$ maps show a positive gradient with the distance to the QSO, hinting at a non-homogeneous distribution of the ionization parameter.
\end{abstract}

Key words: intergalactic medium - quasars: emission lines - quasars: general - quasars: individual (SDSS J095253.83+011421.9) - techniques: imaging spectroscopy

\section{Introduction}

Hall et al. (2004, henceforth H04) published the discovery of an exotic $z=3.02$ quasar (or quasi-stellar object, QSO), SDSS J095253.83+011421.9 (hereafter J0952+0114) lacking a broad Ly $\alpha$ emission line.

H04 invoked several models to explain the observed properties of the emission lines, including dust extinction in the broad-line region (BLR), anisotropic Ly $\alpha$ emission, unusual physical conditions of the BLR (high ionization parameters and a peculiar configuration of the emitting clouds), intrinsic moderate absorption by the resonant transition of $\mathrm{N} \mathrm{V}$ (in the red wing) and $\operatorname{Ly} \alpha$ (in the blue one) to the total $\operatorname{Ly} \alpha$ emission (for a detailed discussion see Section 3 in H04). Finally, they concluded that the dominant effect responsible for the broad Ly $\alpha$ weakness is the presence of very dense gas $\left(n_{\mathrm{H}}=10^{15} \mathrm{~cm}^{-3}\right)$ that is suppressing the broad-line component as well as increasing the collisionally excited metal-line emission.

The partially unsatisfactory interpretation of such peculiar spectral properties has intrigued the community for more than a

\footnotetext{
* Based on observations obtained at the Very Large Telescope (VLT) of the European Southern Observatory, Paranal, Chile (ESO Programme ID 096. A-0345).
}

decade and therefore motivated a follow-up using the Baryon Oscillation Spectroscopic Survey (BOSS, Dawson et al. 2013) by Jiang et al. (2016, henceforth J16) to better determine the true line profiles (and possible physical reasons) behind such a unique QSO spectrum.

Taking advantage of both the wider wavelength coverage (3600-10400 $\AA$ ) and higher resolution $(R \sim 2000)$ of BOSS with respect to the data available from the Sloan Digital Sky Survey (SDSS-I/II) (York et al. 2000), J16 detected the clear imprints of a proximate damped Ly $\alpha$ absorption (PDLA) system with high column density at $z_{\mathrm{abs}}=3.01$ along the line of sight of this QSO (from the damped Ly $\beta$ absorption, the high-order Lyman-series absorptions, the Lyman limit absorption edge, and metal absorption lines). As described in J16, this PDLA is responsible for absorbing the intrinsic Ly $\alpha$ emission of the QSO, while the observed residual narrow Ly $\alpha$ emission line $\left(\mathrm{FWHM} \sim 1000 \mathrm{~km} \mathrm{~s}^{-1}\right.$ ) arises from gas distributed over a larger area than the size of the cross-section size of the PDLA cloud (and possibly more extended than the narrow line region, NLR). Unfortunately, the Ly $\alpha$ emission was not spatially resolved in their SDSS data, and solely based on the large velocity width of the line and covering factor, J16 suggested that such Ly $\alpha$ emission could originate from outflows driven by the QSO. 

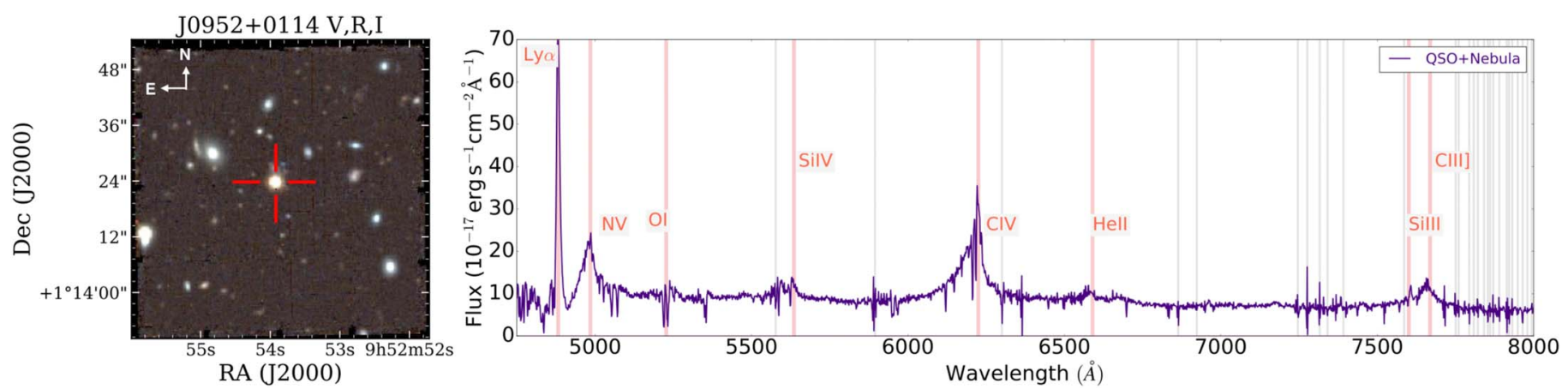

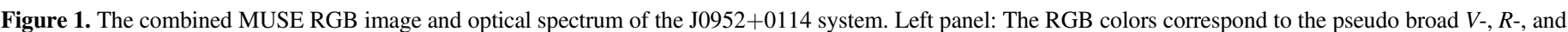

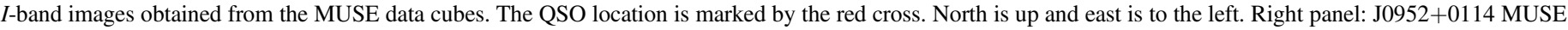

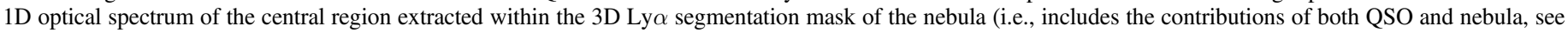
Section 3 for more details). The expected emission lines are labeled in orange and the gray vertical lines indicate the position of the most prominent skylines.

In addition, $\mathrm{J} 16$ found that this system is not so rare, which was also confirmed by Finley et al. (2013) and by the recent works of Fathivavsari et al. (2016, 2018), which identified $\approx 400$ eclipsing damped Ly $\alpha$ absorption systems in the SDSSIII DR12. In particular, Fathivavsari et al. (2018) distinguished three populations of PDLAs based on the strength of the narrow Ly $\alpha$ emission. Their interpretation was that PDLAs result from the interaction between infalling and outflowing gas at different distances from the QSO, supporting the outflow scenario proposed by $\mathrm{J} 16$ for the $\mathrm{J} 0952+0114$ system.

Certainly, as suggested by previous studies, direct observations of extended Ly $\alpha$ emission (the "seeing fuzz" supposed by J16) and detailed kinematic analysis are necessary to test the outflow hypothesis and develop a better understanding of the QSO J0952+0114 and its environment.

In this paper, we will make these steps forward and present the integral field unit (IFU) follow-up observations of J0952 +0114 with the Very Large Telescope (VLT)/Multi Unit Spectroscopic Explorer (MUSE), which have the spatial and spectral domains necessary to provide new insights into the neighborhood of this exotic QSO. The key questions that drive our investigation are: where does the observed narrow $\operatorname{Ly} \alpha$ emission come from? How different (or similar) is this QSO to the other MUSE QSO snapshot (1 hr) fields? Is there any ongoing outflow event in the J0952+0114 system?

We report the detection of extended emission for several lines, including $\mathrm{Ly} \alpha, \mathrm{CIV}$, and $\mathrm{He}$ II, and we investigate possible explanations for the missing broad Ly $\alpha$ in J0952 +0114 and the kinematics of its surrounding gas. Finally, we interpret the possible physical configuration of the different components that are part of the J0952+0114 system (see Figure 10).

The paper is organized as follows. In Section 2 we describe the MUSE observations, data reduction, and post-processing. In Section 3 we detail the analysis performed on the J0952 +0114 data cube. In Section 4 we present our results and in Section 5 we discuss our findings. Finally, we summarize our conclusions in Section 6.

We adopt a flat $\Lambda \mathrm{CDM}$ cosmology with Wilkinson Microwave Anisotropy Probe 9 cosmological parameters of $\Omega_{\Lambda}=0.714, \Omega_{M}=0.286$, and $h=0.693$ (Hinshaw et al. 2013 ), corresponding to $\sim 7.6 \mathrm{kpc} /$ arcsec at redshift $\sim 3$ throughout this work. All wavelengths are specified in vacuum and all magnitudes in the AB system (Oke \& Gunn 1983) unless otherwise stated.

\section{Observations and Data Reduction}

The QSO J0952+0114 has an estimated systemic redshift of $z \approx 3.020 \pm 0.005$ in the literature obtained from several narrow emission lines detected in both the SDSS and BOSS (H04, J16) spectra. Indeed, J0952+0114 exhibits unusual spectral properties with both broad and narrow metal-line emissions but only narrow Ly $\alpha$ emission, as also shown by the MUSE integrated spectrum in Figure 1.

With its I magnitude of 18.95 (Schneider et al. 2003), the quasar is classified as a radio-quiet QSO by Ivezić et al. (2002) because of its radio-loudness parameter $R_{i}<1.024$.

Our MUSE observations of J0952+0114 were carried out in 2016 March as part of our Guaranteed Time Observation (GTO) program, in wide field mode (WFM). MUSE (Bacon et al. 2010) is the integral-field spectrograph mounted on UT4 at the VLT in Paranal, Chile. MUSE combines a relatively large field of view $\left(\approx 1^{\prime} \times 1^{\prime}\right.$ in WFM), an excellent spatial sampling $(0$ ". $2 \times 0$ "! 2$)$, and spectral resolution from 4750 to $9300 \AA$ ( $R$ from $\sim 1750$ to $\sim 3500)$.

We observed the source at the position of $\alpha_{(J 2000)}=$ 09:52:53.8 and $\delta_{(J 2000)}=+01: 14: 22$ for a total integration time of $1 \mathrm{hr}$ under photometric conditions and a point-spread function (PSF) with FWHM of $\sim 0$ !! 7 measured at $7000 \AA$. The observations of QSO J0952+0114 were distributed in four exposures of 15 minutes each, with a dithering pattern smaller than $1^{\prime \prime}$ and a rotation scheme of $90^{\circ}$ for each individual exposure (see also Borisova et al. 2016; Marino et al. 2018, B16 and M18 hereafter, for more details on the data acquisition strategy).

The reduction of the $\mathrm{J} 0952+0114$ data comprises a combination of recipes from the standard ESO MUSE Data Reduction Software (DRS, pipeline version 1.6, Weilbacher 2015) and from CubExtractor software package (CubEx in brief, S. Cantalupo 2019, in preparation; the reader is referred to B16, M18; Arrigoni Battaia et al. 2019; Cantalupo et al. 2019-hereafter A19 and C19-for a description). We employed MUSE scibasic and MUSE scipost routines to perform standard calibration steps on the raw data: master bias, (initial) master flat-fielding, twilight, illumination corrections; wavelength and flux calibrations. The data cubes for each exposure were reconstructed using the geometry and astrometry tables to a common $3 \mathrm{D}$ grid with a sampling of 0 " $2 \times 0 . \prime 2 \times 1.25 \AA$. Subsequently, after refining the astrometry solution with a custom Python script, we improved the 
pipeline flat-field correction using the self-calibrating approach of the CubeFix routine, part of the CubEx package.

Then, we performed the (flux-conserving) sky subtraction using the CubEx routine CubeSharp. These steps (CubeFix and CubeSharp) were repeated twice to minimize possible contamination by continuum sources. Finally, we employed a $3 \sigma$ clipping algorithm to obtain the final average-combined J0952+0114 data cube with CubeCombine (see also B16, M18, A19, and C19 for further details).

The MUSE-reconstructed RGB image of the J0952+0114 field is shown in Figure 1, where the RGB channels correspond to the pseudo broad $V-, R$-, and $I$-band images. The position of the QSO is indicated by the red cross. In Figure 1 we also present the MUSE J0952+0114 spectrum.

\section{Detection of the Spatially Extended Emission}

The main aim of our analysis is to exploit the MUSE IFU's capabilities in characterizing the extended emission originating from the gas surrounding J0952+0114. The standard approach to isolate this "pure" extended emission from that coming from the QSO is to (1) remove the QSO contribution (PSF subtraction) using the CubePSFSub routine part of the CubEx software, and (2) remove contaminating background/ foreground continuum sources using CubeBKGSub. This standard approach, which we used for the He II and C IV emission lines, is described in more detail in B16, M18, and $\mathrm{C} 19$. We note that, in the case of the central Ly $\alpha$ emission for $\mathrm{J} 0952+0114$, the QSO Ly $\alpha$ emission is suppressed by the PDLA, and therefore the QSO PSF subtraction may not be necessary for this line.

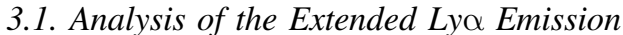

We first tried to quantify the QSO contribution to the central Ly $\alpha$ emission considering the presence of both the peculiar shape of the narrow Ly $\alpha$ emission line and the PDLA. Due to the asymmetric profile and resonant nature of the $\operatorname{Ly} \alpha$ line, performing any line fitting would not help in distinguishing between the contribution of the QSO and that of the nebula to the integrated Ly $\alpha$ line flux. Therefore, we examined the Ly $\alpha$ surface brightness (SB) profile obtained from the continuumsubtracted but not PSF-subtracted Ly $\alpha$ narrowband (NB) image. In Figure 2, the observed azimuthally averaged Ly $\alpha$ $\mathrm{SB}$ profile is shown in blue and the continuum profile in purple. We computed the Ly $\alpha$ SB profile from the pseudo-NB images by summing $\approx 30 \AA$ of the MUSE data cube in the wavelength dimension centered on the QSO $\operatorname{Ly} \alpha$ emission. We also computed the continuum SB profile from the same MUSE data cube by integrating over $400 \AA$ redward of the N V $\lambda 1243$ line and rescaling by a factor of 40 to match the central NB SB profile. Then, we performed both an exponential fit and a power-law fit to the $\operatorname{Ly} \alpha \mathrm{SB}$ profile, considering up to a radius of 100 physical kpc (pkpc) and excluding the most internal part $(<3 \mathrm{pkpc})$ close to the QSO position. We found that the Ly $\alpha$ profile is well traced by a power law (with a slope $\alpha=-2.4$, green solid line in Figure 2), suggesting that, if extrapolated to internal regions, the contribution of the extended nebula to the $\operatorname{Ly} \alpha$ flux in the central region is substantial. Therefore, the Ly $\alpha$ emission could be dominated by the nebula even in the region close to the QSO, as also suggested by the relative excess of Ly $\alpha$ flux with respect to the continuum profile observed at the very smallest radii (beyond $r=3 \mathrm{kpc}$ ) and by the additional

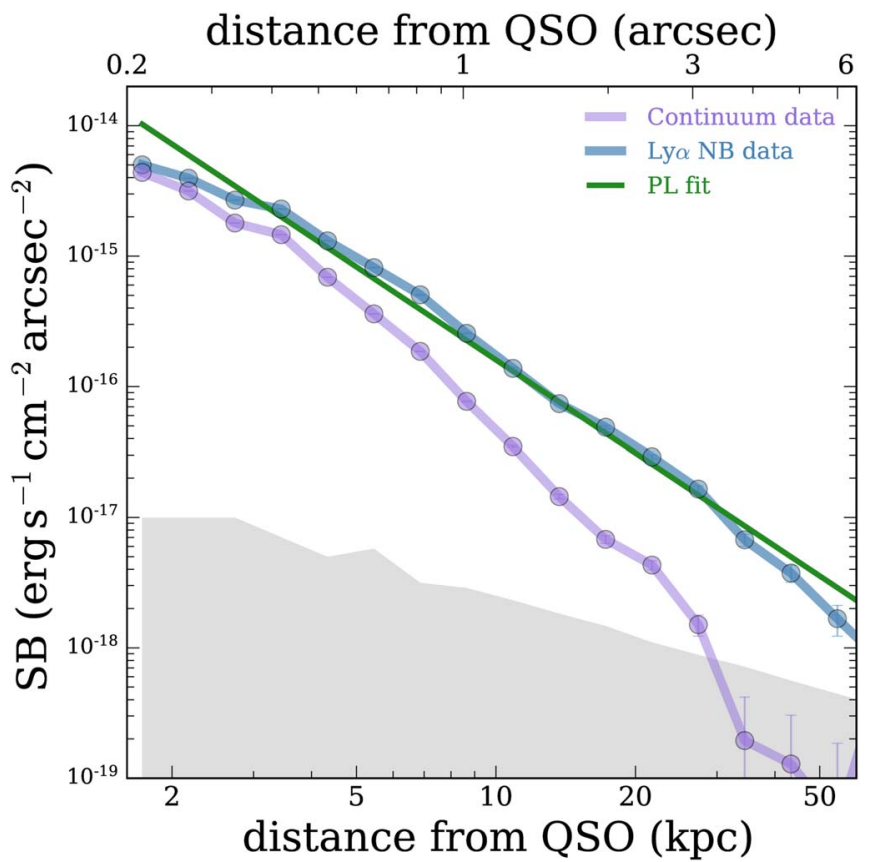

Figure 2. Ly $\alpha$ surface brightness (SB) profiles derived from the pseudo narrowband (NB) Ly $\alpha$ image (only continuum subtracted) and continuum MUSE image. The circularly averaged profile of the continuum light is plotted in purple (solid line and circles) and the Ly $\alpha$ measurements in blue. The green line represents the power-law fit performed to the Ly $\alpha$ SB profile and the gray shaded area shows an estimate of the $2 \sigma$ Gaussian noise associated with the Ly $\alpha$ SB profile.

fact that the Ly $\alpha$ velocity map shows no feature at the center (see Figure 4). This is consistent with the PDLA absorbing all, or almost all, of the intrinsic broad $\operatorname{Ly} \alpha$ emission from the quasar. We speculate that this may be the first case where the emission from a giant Ly $\alpha$ nebula can be traced to such small radii, thanks to the serendipitous help of a natural coronagraph.

Consequently, in the remaining analysis of the Ly $\alpha$ emission we neglect the contribution of the QSO (i.e., a PSF subtraction is not necessary) and only consider the continuum subtraction.

\subsection{The CIV $\lambda 1550$ and He II $\lambda 1640$ Emission Line Detections}

In the case of the other emission lines (C IV and He II), due to the clear broad component identified in the integrated spectrum (see Figure 1), we followed the classical analysis procedure that includes both empirical PSF and continuum subtraction as already done in B16. Briefly, our empirical PSF modeling is based on the estimation of the QSO flux in the central area of $1^{\prime \prime} \times 1^{\prime \prime}$ (or $5 \times 5$ pixels $^{2}$, assuming that the central flux budget is dominated by the QSO) on pseudo-NB images of $187 \AA$ wide (150 pixels in the spectral dimension). Then, each reconstructed PSF image is rescaled using an averaged-sigma-clip algorithm and subtracted from the corresponding wavelength layer. After masking the spectral layers associated with the nebulae plus any continuum objects around the QSO, we iteratively ran CubePSESub, producing accurate results on large scales around the QSO, as already successfully applied in B16, North et al. (2017), and Ginolfi et al. (2018), among others.

\subsection{Extracting the Extended Emission Lines in $3 D$}

Subsequently, we subtracted the continuum using CubeBKGSub based on a fast median-filtering approach with 

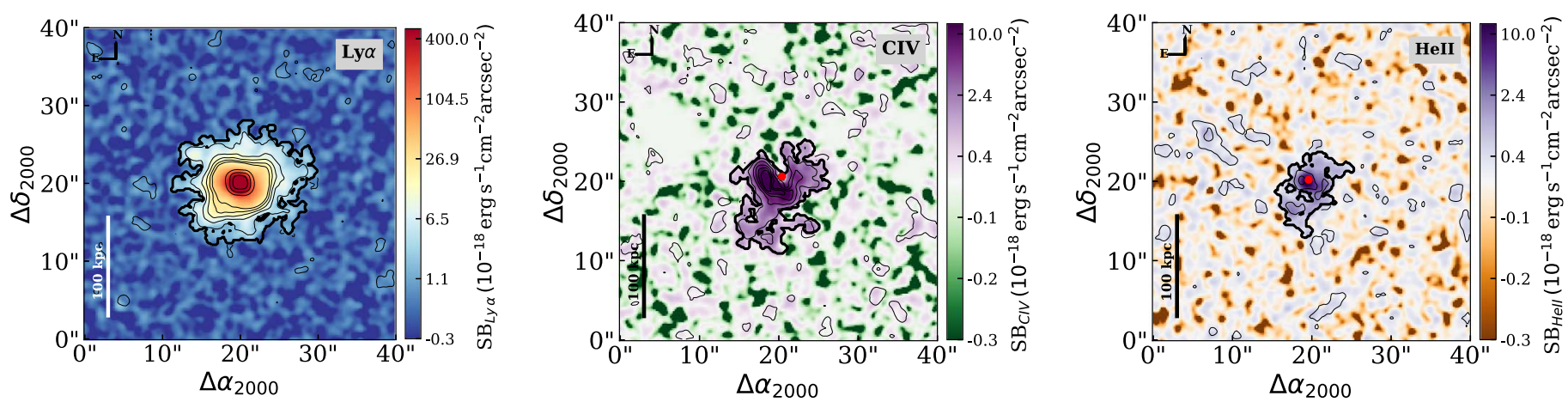

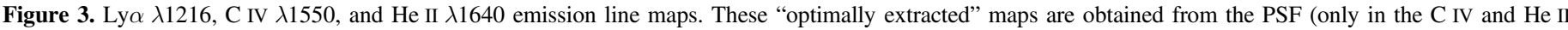

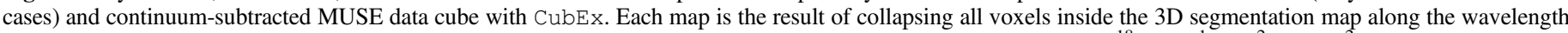

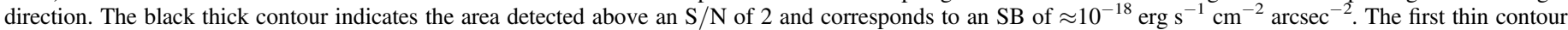

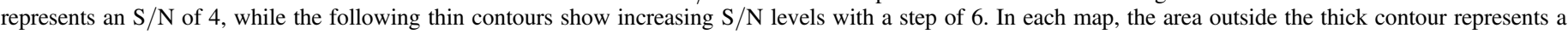

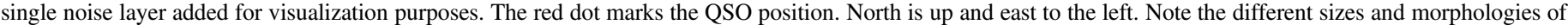
these emission line nebulae.
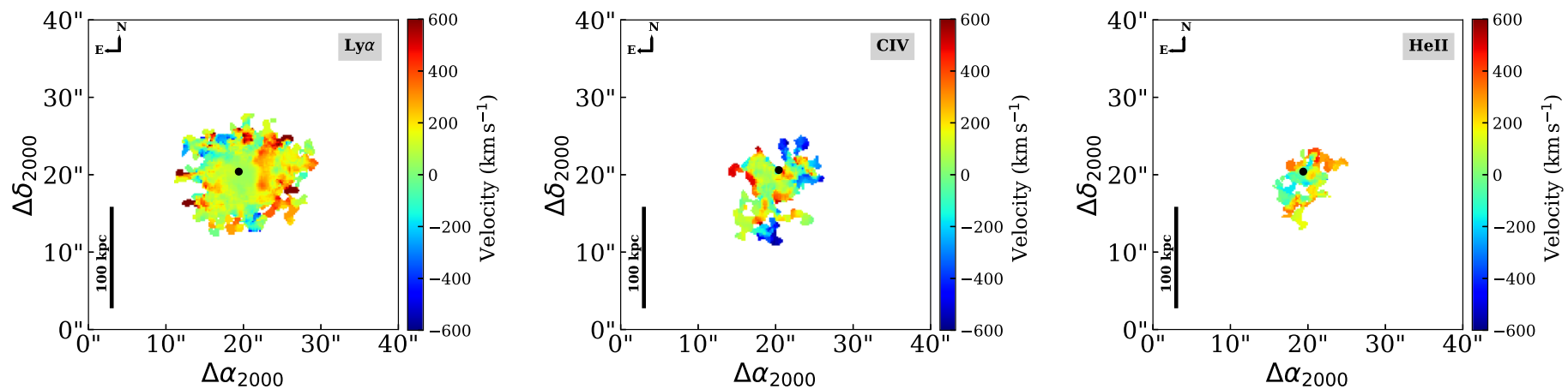

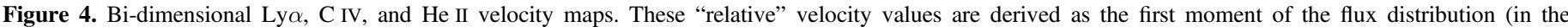

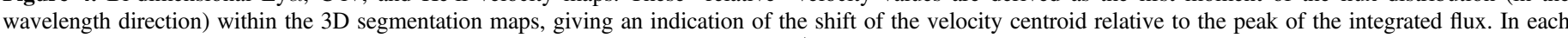

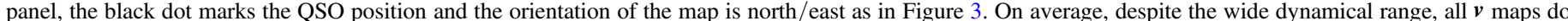

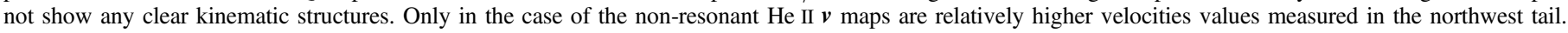

the most prominent emission lines masked. We optimized this procedure for each emission line. In particular, we created three different post-processed versions of the data cube around the most prominent emission lines: $\mathrm{Ly} \alpha, \mathrm{C} \mathrm{IV}$, and He II.

We selected post-processed sub-cubes $(80 \AA$ or 100 wavelength pixels wide) centered at the expected emission wavelength, using as initial guess a QSO systemic redshift of 3.02, and searched for any possible extended emission. We then ran CubEx for object extraction and detection directly on the data cube above a user-defined signal-to-noise ratio $(\mathrm{S} / \mathrm{N})$ threshold, in both the spatial and spectral dimensions. In order to perform a consistent comparison between the different emission lines, we used CubEx with the most general input parameters as follows: (i) (Gaussian) spatial filtering of 0!" 4 (no smoothing in the wavelength direction); (ii) spatial $\mathrm{S} / \mathrm{N}$ of 2 (equivalent to an SB of $\approx 10^{-18} \mathrm{erg} \mathrm{s}^{-1} \mathrm{~cm}^{-2} \operatorname{arcsec}^{-2}$ at $1.25 \AA$ ) with a minimum of connected voxels ${ }^{10}$ of 1000 (after spatial smoothing); (iii) rescaled variance; (iv) masking of bright continuum sources and sky residuals. In addition to Ly $\alpha \lambda 1216$, we extracted both extended C IV $\lambda 1550$ and He II $\lambda 1640$ emission lines. The 2D projections of the flux (Figure 3), velocity (Figure 4), and velocity dispersion (Figure 5) are based on the three-dimensional segmentation mask (3D mask in short) obtained from this extraction procedure (see also B16 for further details). Table 1

\footnotetext{
${ }^{10}$ Spatial and spectral elements in the MUSE data cube.
}

summarizes the observed and derived physical properties for each emission line. The observed flux-weighted Ly $\alpha$ wavelength, $4882.0 \AA$, corresponds to a redshift $z=3.0176$.

\subsection{Analysis of the PDLA Emission Features}

Previous studies ( $\mathrm{H} 04$ and J16) confirmed the presence of a PDLA in the line of sight of the QSO J0952+0114, making use of the bluer wavelength coverage of the SDSS and BOSS spectra with respect to our MUSE data set. From the analysis of the absorption lines (from Ly $\beta$ to Ly9) identified in the BOSS spectrum between 3600 and $4400 \AA$, J16 inferred a redshift of $z_{\mathrm{abs}}=3.01$ for the PDLA. Therefore, we analyzed our MUSE spectrum in order to find any possible emission and absorption features at the PDLA redshift. We caution that, due to the small differences in redshift between the nebula (QSO) and the PDLA, $\Delta z=0.007(\Delta z=0.01)$, and the higher intrinsic luminosity of the nebula (QSO) emission lines with respect to the PDLA ones, possibly some of the components may be blended. We identified the PDLA emission lines in the continuum-subtracted spectrum extracted from a region with a diameter of 0 " 6 centered on the QSO. We found a clear detection of isolated He II emission at the PDLA redshift (see Figure 6). We also note that for the C IV doublet lines there is a clear absorption system corresponding to the PDLA redshift. In the cases of the $\mathrm{N} \mathrm{V}$ and $\mathrm{C} \mathrm{III]} \mathrm{emission} \mathrm{lines,} \mathrm{we} \mathrm{do} \mathrm{observe}$ some emission features but it is very difficult to distinguish 

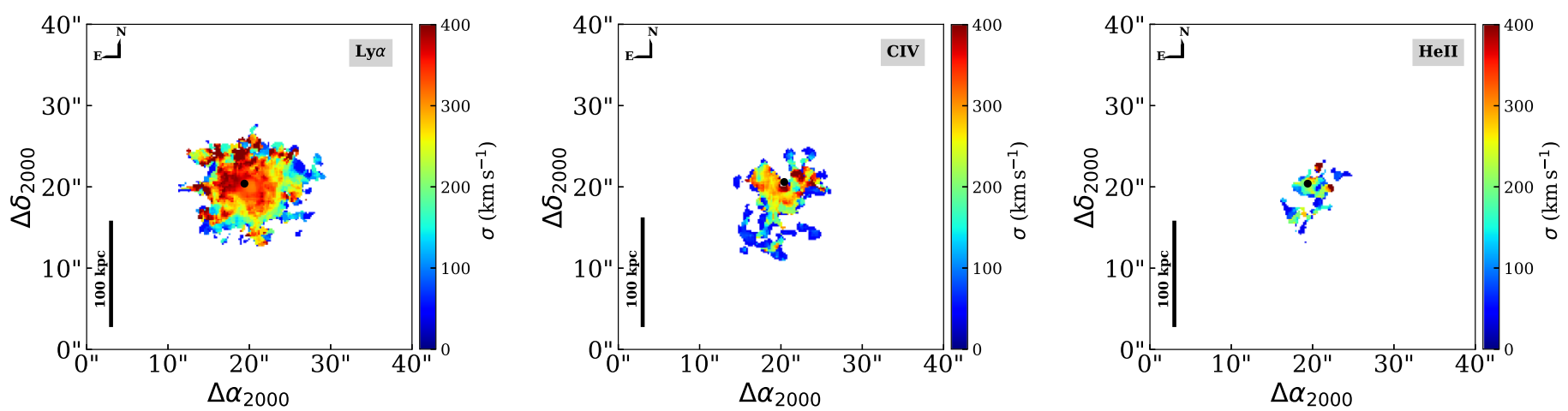

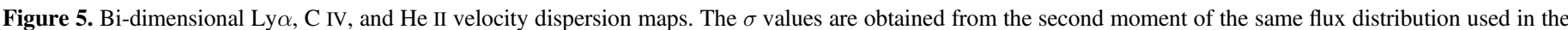

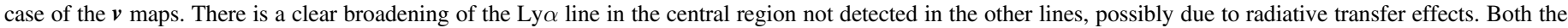

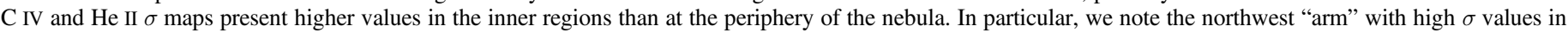
the He II map. As in the previous figure, the QSO position is indicated by the black dot and the maps are oriented north/east.

between the contributions of the PDLA and of the QSO/nebula to the global line profiles (see Figure 11 in the Appendix).

\section{Properties of the Spatially Extended Emission}

Taking advantage of MUSE's wide optical coverage, and after applying the methodology described above, we detected extended emission from the circumgalactic medium (CGM) of $\mathrm{J} 0952+0114$ in the $\operatorname{Ly} \alpha \lambda 1216$, C IV $\lambda 1550$, and He II $\lambda 1640$ emission lines. As described in Section 3, all these lines are detected with an $\mathrm{S} / \mathrm{N}$ greater than 2 (after spatial smoothing per voxel) and they have at least 1000 MUSE voxels connected (see Table 1).

\subsection{Morphology of the Emission Line Nebulae}

The "optimally extracted" (OE) images of the emission line nebulae detected in the $\mathrm{J} 0952+0114$ QSO field at $z=3.02$ are shown in Figure 3. Differently from standard NB images, we computed the OE maps using a differential spectral width for the nebulae and for the noise. By collapsing all the spectral layers detected in the 3D mask, features across a range of spectral widths can be highlighted with little noise. As a visual aid, we include a single layer of noise and the thick black contour in order to give a visual estimation of the noise level of our observed data. Each OE map has a linear size of $40^{\prime \prime}$, where the white (or black) vertical line shows a spatial extent of $100 \mathrm{pkpc}$, and their orientation is north/east with the position of the QSO indicated by the red dot. The spectral width $\left(\Delta \lambda_{\text {size }}\right)$ of this OE map is specified in the fifth column of Table 1 .

It is worth noting that the detected emission lines present a large variety of shapes and sizes. For instance, the Ly $\alpha$ nebula extends for more than $130 \mathrm{pkpc}\left(1^{\prime \prime}\right.$ at $z=3.0176$ corresponds to $7.6 \mathrm{pkpc})$ with a wide spectral width $\left(\Delta \lambda_{\text {size }}=30 \AA\right) .{ }^{11} \mathrm{In}$ addition, the $\operatorname{Ly} \alpha$ nebula has a more circularly symmetric appearance than the CIV and He II nebulae (see Figure 3). The integrated Ly $\alpha$ flux over the $3 \mathrm{D}$ mask is $F(\operatorname{Ly} \alpha) \sim$ $7.4 \times 10^{-15} \mathrm{erg} \mathrm{s}^{-1} \mathrm{~cm}^{-2}$, which, at this distance, corresponds to a luminosity of $\sim 6 \times 10^{44} \mathrm{erg} \mathrm{s}^{-1}$, under the assumption of isotropic emission. Moreover, the C IV OE map (and marginally also the He II OE map) presents a "cavity" in the north direction that is not observed in the case of the $\operatorname{Ly} \alpha$ emission.

\footnotetext{
11 The nebula size is computed as the maximum projected size of the $3 \mathrm{D}$ masks.
}

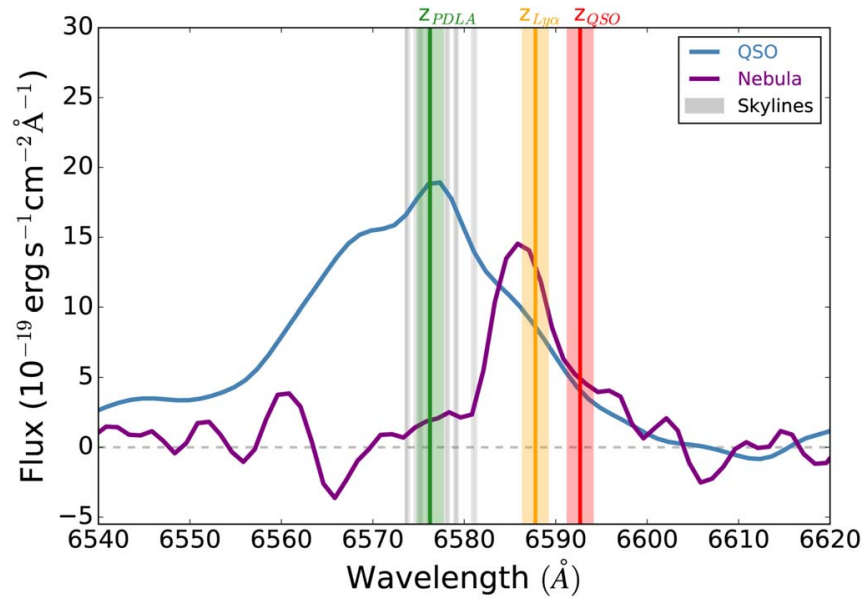

Figure 6. Comparison between the He II emission coming from the QSO (blue line) and and that from the nebula (purple line). The QSO integrated spectrum was obtained from a 0 ." 6 aperture centered on the QSO after the continuum subtraction was performed. The QSO spectrum was rescaled by a factor of 30 and a smoothing of 2 pixels $(2.5 \AA)$ is applied for visualization purposes. In the case of the nebula, the purple spectrum represents the PSF-subtracted He II emission within a $1^{\prime \prime}$ aperture shifted $2^{\prime \prime}$ to the east from the QSO. The green, orange, and red vertical lines indicate the He II emission in the case of adopting the systemic redshift of the PDLA, the Ly $\alpha$, or the QSO, respectively. The gray vertical lines mark the positions of the strongest residual skylines.

The C IV emission map shows evidence of a clear asymmetry and a more filamentary structure with respect to the other emission lines. With a projected size of $\sim 100 \mathrm{pkpc}$ and a total flux $F(\mathrm{CIV}) \sim 5 \times 10^{-16} \mathrm{erg} \mathrm{s}^{-1} \mathrm{~cm}^{-2}$ (i.e., $L(\mathrm{C} \mathrm{IV}) \sim$ $\left.4 \times 10^{43} \mathrm{erg} \mathrm{s}^{-1}\right)$, it looks particularly elongated in the south direction, while in the north the aforementioned cavity is very prominent. The He II extended emission map, with a flux $F($ He II $) \sim 9 \times 10^{-17} \mathrm{erg} \mathrm{s}^{-1} \mathrm{~cm}^{-2}$, a luminosity of $L(\mathrm{He}$ II $) \sim$ $8 \times 10^{42} \mathrm{erg} \mathrm{s}^{-1}$, and a size of $\sim 75 \mathrm{pkpc}$, is characterized by a more compact morphology with a central circular component with two "arms" going out in the west and south directions. Finally, we observe small spatial offsets between the peaks of the nebula emission and the QSO position; in particular, the offsets are larger than few arcseconds only in the case of the C IV OE maps. The difference in the morphologies of the emission lines can be partly due to the different intrinsic SB and therefore detection limits of different lines. However, it can also tell us something about intrinsic gradients associated with 
Table 1

Derived Properties of the Spatially Extended and Spectrally Narrow Emission Lines

\begin{tabular}{lccccrr}
\hline \hline Line & \# Connected Voxels & $\begin{array}{c}\lambda_{\text {detected }}^{\mathrm{a}} \\
(\AA)\end{array}$ & $\begin{array}{c}\text { Area }^{\mathrm{b}} \\
\left(\operatorname{arcsec}^{2}\right)\end{array}$ & $\begin{array}{c}\Delta \lambda^{\mathrm{c}} \\
(\AA)\end{array}$ & $\begin{array}{c}\text { Flux }^{\mathrm{d}} \\
\left(10^{-17} \mathrm{erg} \mathrm{s}^{-1} \mathrm{~cm}^{-2}\right)\end{array}$ & $\begin{array}{c}L^{\mathrm{e}} \\
\left(10^{42} \mathrm{erg} \mathrm{s}^{-1}\right)\end{array}$ \\
\hline Ly $\alpha$ & 33009 & 4882.00 & 176 & 37 & $742.8 \pm 0.4$ & 605.8 \\
C IV & 11768 & 6218.95 & 83 & 30 & $46.2 \pm 0.1$ & $274_{-120}^{+366}$ \\
He II & 2386 & 6589.27 & 41 & 19 & $9.6 \pm 0.2$ & $167_{-42}^{+298}$ \\
\hline
\end{tabular}

Notes.

${ }^{a}$ Flux-weighted wavelength.

b The area represents the number of pixels inside the 3D mask.

c Spectral width of the 3D mask.

${ }^{d}$ The line flux is computed as the integrated value within the $3 \mathrm{D}$ mask

e The luminosity is derived assuming a redshift of $z=3.02$.

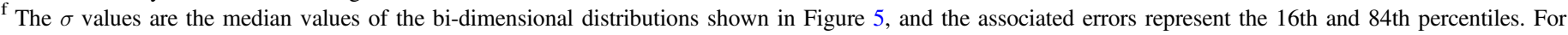
consistency with literature works, the Gaussian-equivalent FWHM can be derived by multiplying $\sigma$ by 2.355 .

different physical conditions of the ionized gas (i.e., ionization parameter) within the nebula.

The He II (and C IV) emission is more elongated than that of Ly $\alpha$. As He II is a non-resonant line and requires photons of energy $\gtrsim 54 \mathrm{eV}$ (to produce doubly ionized $\mathrm{He}$ that then recombines to $\mathrm{He}$ II), its morphology better traces the regions with the high ionization levels. If this is true, the gas within the elongated He II structure either has a lower density than the surroundings or it is subject to a more intense ionizing radiation field. The latter option, in particular, could be an indication that the bright He II emission traces, to some extent, the ionization cone of the QSO. A similar explanation may also hold for the morphology of CIV, which is also bright in the region orthogonal to the main emission "cone" of the QSO as possibly traced by high-ionization non-resonant lines (i.e., He II).

\subsection{Lyo, CIV, and He II Kinematics}

The resolved kinematic information is summarized in Figures 4 and 5, where we report maps of the velocity, $v$, and the velocity dispersion, $\sigma$, respectively, in the case of the three extended emission lines detected in the J0952+0114 QSO field, i.e., Ly $\alpha, \mathrm{CIV}$, and He II. The goal here is to identify any possible kinematical features, such as rotation, inflow, outflow, or some special kinematic region within the nebulae. In particular, from the information given by the joint analysis of the 2D $v$ and $\sigma$ maps for the three emission lines, the intriguing question hypothesized by $\mathrm{J} 16$ on whether there is evidence of an outflow at large radii from the QSO can be addressed. It is well known that the Ly $\alpha$ line is not the best kinematic tracer given its resonant nature, and some caution also has to be taken when deriving conclusions from the C IV line, which is also resonant. As mentioned in Section 3, we made use of the 3D segmentation masks to derive the first and second moments of the flux distribution (i.e., we do not perform any fit to the emission lines), which give us an idea of both the velocity centroid and shift with respect to the systemic redshift of the nebula $(z \sim 3.017)$ computed from the fluxweighted Ly $\alpha$ wavelength (see Table 1). We used this redshift as a reference velocity for the other emission lines.

In general, we find that the dynamical range covered by the three lines in both $v$ and $\sigma$ maps is in agreement with similar ranges derived from other giant Ly $\alpha$ nebulae detected around typical bright QSOs without PDLAs. For instance, $\sigma_{\mathrm{Ly} \alpha}$ is similar to the values measured by B16 and A19. In the case of the Ly $\alpha \boldsymbol{v}$ map of the nebula, we noticed some high-velocity structure in the west direction. In the case of the C IV $v$ map no clear kinematic patterns are found except for the lower velocity values in the periphery of the filamentary structure. Obviously, however, interpreting the Ly $\alpha$ line width in terms of intrinsic kinematics is not always an easy task, because the Ly $\alpha$ line can also be significantly broadened by radiative transfer effects.

Moreover, the He II $v$ map shows a mildly coherent velocity gradient, with the arms (particularly the northwestern one) having higher values than the central region. Due to the poorer $\mathrm{S} / \mathrm{N}$ of the arms with respect to the central region, we would require deeper data to be able to confirm this result. The velocity dispersion maps, shown in Figure 5, look very different in the cases of Ly $\alpha, \mathrm{C}$ IV, and He II. A clear structure with high velocity dispersion is detected in the Ly $\alpha$ and C IV $\sigma$ maps in the central region, while the C IV and He II $\sigma$ maps show a gradient with higher values in the center that decreases with increasing distance from the QSO.

Thanks to our MUSE data, on the one hand, we can confirm a fairly high $\sigma_{\mathrm{Ly} \alpha} \simeq 300 \mathrm{~km} \mathrm{~s}^{-1}$ as representative of a large portion of the nebula, even at spatial scales much larger than those observationally probed by J16. On the other hand, however, the velocity dispersion of the narrow non-resonant He II line, which we can more safely take as indicative of the intrinsic dispersion of the cold CGM gas, is $\sigma_{\mathrm{He} \mathrm{II}} \sim 150 \mathrm{~km} \mathrm{~s}^{-1}$ (see Figure 5 and Table 1). This is only half of $\sigma_{\mathrm{Ly} \alpha}$ and smaller than the expected escape velocity. We conclude that the relatively large value of $\sigma_{\text {Ly } \alpha}$ is likely not intrinsic and rather mostly a consequence of radiative transfer (Neufeld 1991; Dijkstra et al. 2006).

The velocity gradient in the He II velocity map may also be interpreted as being composed of two velocity structures, one in the center at lower velocities and the two faster arm regions. Due to the significant heterogeneity and asymmetry of the velocity field, we rule out ordered rotation as a likely explanation for the observed kinematic pattern.

Therefore, we conclude that we are most likely witnessing a significant gas circulation within the CGM of the galaxy hosting J0952+0114. However, we do not find convincing evidence for a strong outflow that is able to escape from the CGM in the central region from the He II $\boldsymbol{v}$ and $\sigma$ maps (note also that the values in the very central region should be interpreted with caution due to possible effects from PSF subtraction).

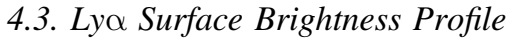

In Figure 2 we show the Ly $\alpha \mathrm{SB}$ profile of the nebula enshrouding the QSO J0952+0114. The PDLA blocks the 


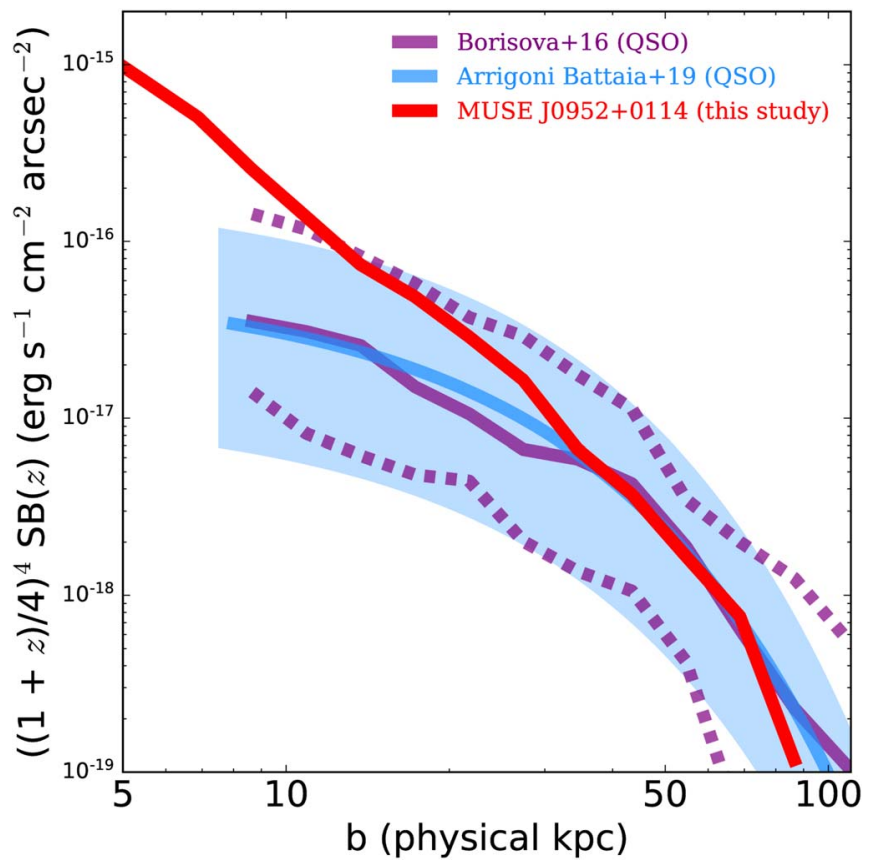

Figure 7. Ly $\alpha$ surface brightness (SB) profile of the J0952+0114 nebula as a function of the projected physical distance from the QSO. The circularly averaged profile is plotted with the red solid line. Other Ly $\alpha$ SB profiles are also plotted for comparison. In particular, the median SB profile of the MUSE Ly $\alpha$ nebulae from B16 is shown in purple with the dashed lines marking the 10th and 90th percentiles. This median profile has been rescaled to $z=3$. The best exponential fit (and its dispersion) of the SB profiles from the QSO MUSEUM is plotted with the solid blue line (light blue area, Arrigoni Battaia et al. 2019).

QSO emission, and the extended nebula dominates the total Ly $\alpha$ emission even at small radii. Moreover, one of our objectives is to understand how the $\mathrm{J} 0952+0114$ Ly $\alpha$ nebula compares to other radio-quiet QSOs observed with MUSE. In order to contextualize this nebula, we measured the SB Ly $\alpha$ profile through the standard NB images, similar to previous works (B16, A19). We computed the circularly averaged profile from the pseudo-NB image obtained by collapsing the MUSE data cube using the maximum spectral size of the 3D mask as the width, which in the case of our Ly $\alpha$ emission is $30 \AA$. We then performed an exponential fit and a power-law fit above the $2 \sigma$ noise limit, i.e., $\mathrm{SB}$ of $\approx 0.5 \times 10^{-18} \mathrm{erg} \mathrm{s}^{-1}$ $\mathrm{cm}^{-2} \operatorname{arcsec}^{-2}$, that returns a normalization parameter (at a radius of $10 \mathrm{pkpc}$ ) of $\log _{10}\left(C_{p}, r_{10}\right)=-15.8$ and a slope $\alpha$ of -2.4, compatible with other Ly $\alpha$ SB profiles obtained with MUSE. More details on the fitting process are provided in Appendix B of B16. The red line in Figure 7 shows the circularly averaged values computed in units of $\mathrm{SB} \times((1+$ $z) / 4)^{4}$ with an extent of more than $100 \mathrm{pkpc}$ from the QSO. We chose these units because we wanted to perform a consistent comparison of the J0952+0114 SB profile with the other Ly $\alpha$ systems in the literature and therefore avoid any redshift dependence and cosmological dimming effects. Figure 7 also shows the comparison with previous works as the median profile of the MUSE QSO nebulae we published in Borisova et al. 2016 (purple line, see also Appendix C) and the best exponential fit to the QSO MUSEUM sample (A19, blue line). As discussed later in Section 5, the outer parts (beyond $10 \mathrm{pkpc}$ ) of the J0952+0114 SB profile are very similar to what we found in other giant fluorescent nebulae around QSOs at $z \approx 3$. The innermost regions are brighter due to the presence of

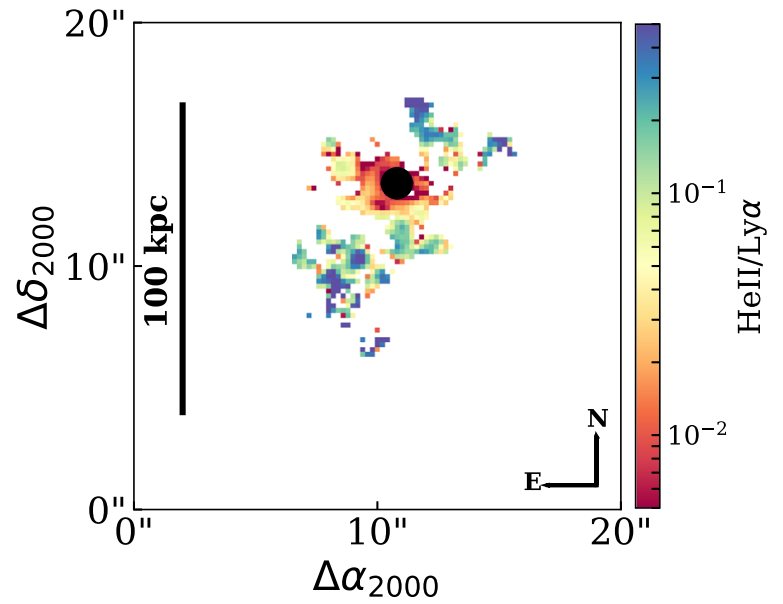

Figure 8. Bi-dimensional map of $\mathrm{He}$ II $/ \mathrm{Ly} \alpha$ ratio. The ratio values are derived in the Ly $\alpha, \mathrm{C}$ IV, and He II common spatial mask (see text for details) from the smoothed (Gaussian smoothing with a radius of 2 spatial pixels) pseudo-NB images.

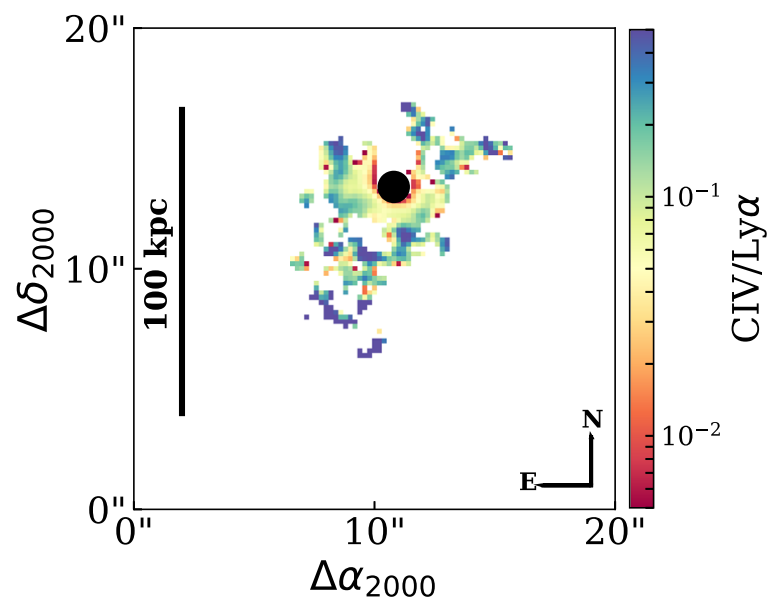

Figure 9. Bi-dimensional map of $\mathrm{C} \mathrm{IV} / \mathrm{Ly} \alpha$ ratio. As in Figure 8, the line ratio is obtained from the smoothed C IV and Ly $\alpha$ pseudo-NB images.

the PDLA; however, this could reflect the difficulty of tracing the nebula into the center of typical objects (without PDLAs).

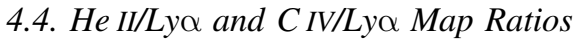

Ly $\alpha \lambda 1216$, C IV $\lambda 1550$, and He II $\lambda 1640$ are some of the brightest rest-frame UV lines employed to better constrain the nature of ionized gas in emission. In particular, the ratios of these lines, i.e., $\mathrm{He} \mathrm{II} / \mathrm{Ly} \alpha$ and $\mathrm{CIV} / \mathrm{Ly} \alpha$, are particularly useful for insights into the physical properties of the emitting gas, such as the ionization parameter, density, and metallicity (Villar-Martin et al. 1997; Arrigoni Battaia et al. 2015; Prescott et al. 2015; C19 and references therein).

Differently from previous works, in the case of the gas surrounding $\mathrm{J} 0952+0114$, we were able to measure the spatial distribution of these line ratios instead of using limits. The resulting maps of emission line ratio are presented in Figures 8 and 9. In order to avoid any aperture effects in the line ratios, we derived these maps with an intersection of the 3D segmentation masks (where we simultaneously detected the three emission lines) and smoothing of 2 spatial pixels (radius) for display purposes.

The J0952+0114 field is a rare case among giant Ly $\alpha$ nebulae around high-redshift QSOs in which these ratios can be 
measured in a consistent way and spatially resolved on extended scales. In general, the bi-dimensional distributions of $\mathrm{He}$ II/Ly $\alpha$ and $\mathrm{CIV} / \mathrm{Ly} \alpha$ line ratios are not homogeneous, as also suggested by previous observations and theoretical works (C19, Corlies et al. 2018; McCourt et al. 2018, among others). Particularly, ratios in the inner region are lower and more ordered than in the outer parts, where these values tend to be relatively higher and less spatially structured. In particular, using a $2^{\prime \prime}$ region centered on the QSO, we measure a median $\mathrm{He}$ II $/ \mathrm{Ly} \alpha$ ratio of 0.01 and a $\mathrm{CIV} / \mathrm{Ly} \alpha$ ratio of 0.05 . If we move the same aperture in the outer part 1 ." $^{\prime \prime}$ to the east and $4^{\prime \prime}$ to the south of the QSO position, we obtain 0.09 and 0.11 , respectively. In addition, the integrated values for both emission line ratios, measured over the entire maps, are $\mathrm{He} \mathrm{II} / \mathrm{Ly} \alpha=0.03$ and $\mathrm{C} \mathrm{IV} / \mathrm{Ly} \alpha=0.09$, in agreement with previous MUSE studies of radio-quiet QSOs (see Figure 8 in B16). Finally, the dynamical range covered by the resolved ratio maps confirms that the $2 \sigma$-limit values used in B16 were very conservative with respect to the detected values, being a factor of two lower than the integrated measured ratios. This implies that the $\mathrm{J} 0952+0114$ nebula presents consistent emission line ratios compared to the MUSE radio-quiet nebula and is also compatible with some of the radio-galaxy halos plotted in Figure 8 of B16.

The very low values of He II/Ly $\alpha$ observed in the central region of the $\mathrm{J} 0952+0114$ nebula may be due to the fact that a significant fraction of the observed $\operatorname{Ly} \alpha$ emission in these regions is not produced locally by recombination but is due to scattering. We note, however, that, even in the most illuminated region, the observed $\mathrm{He} \mathrm{II} / \mathrm{Ly} \alpha$ ratio is slightly larger, but still about a factor of 2 below the expectation for pure recombination in a low-density medium. This may imply that scattering effects are non-negligible in this region as well. At larger distances, the effect of the Ly $\alpha$ scattering should diminish, due to the fact that the optical depth to $\operatorname{Ly} \alpha$ scattering can become sufficiently large and recombination radiation is expected to be the dominant emission mechanism (as in the case of the Slug nebula detailed in Leibler et al. 2018). This would explain the higher line ratios measured in the outer regions. Alternatively, the observed $\mathrm{He}$ II $/ \mathrm{Ly} \alpha$ ratio could be an indication of the presence of unresolved high-density clumps or of a broad density distribution of the cold photoionized gas; this, in fact, has been proposed as a mechanism to explain low He II/Ly $\alpha$ ratio in recombination-dominated regions of the CGM around QSOs. Indeed, at fixed average density, if the full density distribution is broader than a delta function, then some fraction of the gas can have a sufficiently large density that the helium is not completely ionized, with a consequent drop in the emissivity of the He II $\lambda 1640$ recombination line (see C19 for a more thorough discussion of this point).

With these caveats in mind, it is interesting to notice that the size of the observed nebula is up to one order of magnitude larger than those of the NLR models in Feltre et al. (2016), so a first-order (admittedly simplistic) scaling based on the ionization parameter would suggest densities down to two orders of magnitudes smaller than those of Feltre et al. (2016), $n_{e} \lesssim 1-10 \mathrm{~cm}^{-3}$, consistent with diffuse medium. Note that the simple scaling based on ionization parameter is a very crude approximation and that tailored photoionization models, considering the spatial dependence of the line ratios and radiative transfer effects, would be necessary to come to more secure conclusions.

\section{Discussion}

The new MUSE observations, described in Section 4, add further, important elements to our understanding of the "exotic" quasar J0952+0114:

(i) the quasar is surrounded by a giant $\operatorname{Ly} \alpha$ nebula with $\mathrm{SB}$ profile and morphology similar to other nebulae discovered with MUSE (Figure 3);

(ii) the central Ly $\alpha$ emission in the spectrum of J0952+0114 is consistent with the extrapolation of the SB profile of the giant nebula (Figure 7);

(iii) there are at least two components of He II emission in the central part-a broader component at the redshift of the PDLA and a narrow component at the corresponding redshift of the Ly $\alpha$ emission (Figure 6).

In this section, we combine these elements with previous results and discuss the most likely scenarios for the origins of both the PDLA and extended emission around J0952+0114. Finally, we put these results into a broader context with a particular focus on outflows from active galactic nuclei (AGNs).

\subsection{Origin and Location of the PDLA}

The lack of observable Ly $\alpha$ emission from the BLR, as already argued by previous studies, implies that the PDLA should be at least as large as the Ly $\alpha$ BLR of the quasar itself. Recent reverberation mapping studies have found that BLR Ly $\alpha$ and C IV emissions typically originate from similar regions (Lira et al. 2018). Since the C IV from the BLR is visible in the spectrum of the quasar, this is suggesting either that the PDLA gas is not very optically thick to C IV $\lambda 1550$ (because of low metallicity or lower/higher ionization state) or that the part of the PDLA optically thick to C IV is not fully covering the $\mathrm{C}$ IV BLR, providing therefore a possible upper limit on the size of the PDLA. The latter hypothesis would require the Ly $\alpha$-emitting BLR to be much smaller than the C IV one, which is in contradiction to the reverberation mapping results. Therefore, the analysis of the quasar spectrum alone does not provide clear information on the size and spatial distribution of the gas associated with the PDLA.

Although indirectly, the presence and properties of the giant Ly $\alpha$ nebula discovered around $\mathrm{J} 0952+0114$ provide a constraint on the "illumination" from the BLR on a much larger solid angle than our line of sight. In particular, this applies to both the cases of fluorescent recombination and BLR Ly $\alpha$ scattering for the origin of the Ly $\alpha$ nebula (see Cantalupo 2017 for a review). Therefore, the presence of extended properties, i.e., the nebula, already excludes the scenario in which the PDLA fully covers the emitting solid angle of the BLR. On the contrary, the circular symmetry of the Ly $\alpha$ SB around the quasar seems to indicate that the PDLA coverage is small and either uniform across the emitting solid angle or restricted to a small cone that contains our line of sight below our spatial resolution scale. As shown in Section 4, the giant Ly $\alpha$ nebula around $\mathrm{J} 0952+0114$ shares similar Ly $\alpha$ emission properties with other nebulae detected around QSOs without PDLAs, reinforcing the idea that the majority of the emitting solid angle is visible from the majority of lines of sight through the CGM.

This scenario has two possible implications: (i) the PDLA is an intervening system in the CGM/intergalactic medium (e.g., 


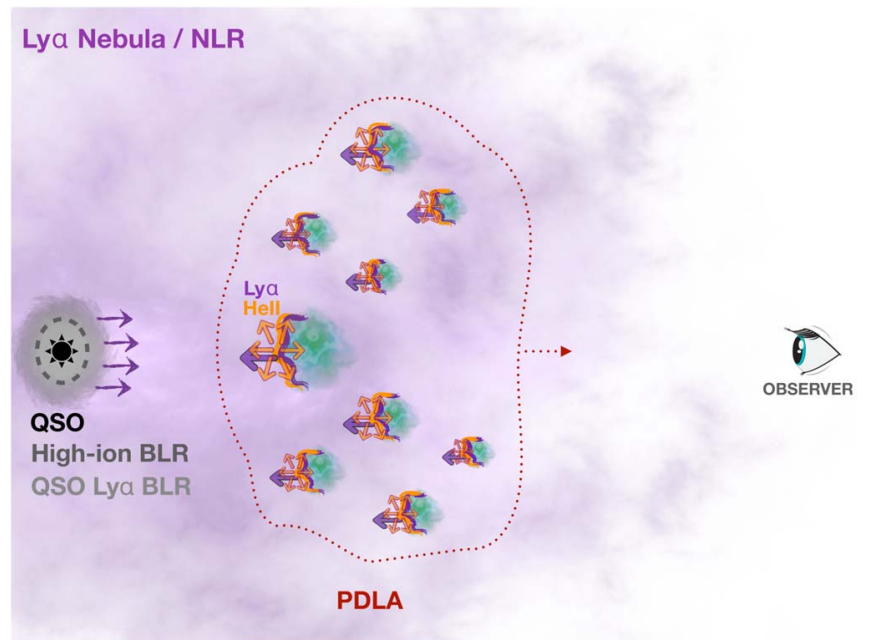

Figure 10. Schematic view of the environment of QSO J0952+0114. The QSO at $z=3.02$ is eclipsed by the PDLA composed of outflowing clouds (detected in absorption at $z=3.01$ ) along the line of sight to the QSO. The Ly $\alpha$ emission from the BLR is emitted and scattered back toward the QSO (purple arrows) while the He II recombination emission, due to its non-resonant nature, is able to pass through the PDLA clouds (orange arrows). The giant Ly $\alpha$ nebula and the NLR (in purple) are surrounding the QSO, the BLR, and the PDLA.

gas associated with a foreground galaxy), not directly associated with the quasar, (ii) the PDLA is intrinsically small and physically proximate to the quasar. Although neither of these two options can be discarded with certainty, we note that the broad He II emission detected along our line of sight has exactly the same redshift as the PDLA (Figure 6). This He II emission is significantly different than what is typically observed in other quasars with similar luminosity, redshift, and UV slope (see Figures 12 and 13) in terms of both equivalent width and shift from other high- and low-ionization lines, suggesting that it could be associated with the PDLA itself. If that is indeed the case, such broad and bright He II emission would require an ionization parameter high enough to imply that the PDLA should be possibly very close to the quasar, i.e., within the NLR, or certainly within the host galaxy. A narrow and fainter He II component spectrally coincident with the extended nebular Ly $\alpha$ emission (and therefore redshifted by $\approx 500 \mathrm{~km} \mathrm{~s}^{-1}$ with respect to the broad He II) is also present close to the center, suggesting the presence of a multi-phase medium with different kinematics and densities. In this scenario, the broad He II component would therefore represent the non-resonant emission produced by a clumpy medium with a bulk velocity of $\approx 500 \mathrm{~km} \mathrm{~s}^{-1}$ with respect to the host galaxy (the systemic redshift of which is taken from the narrow He II component) and a velocity dispersion of $\approx 600 \mathrm{~km} \mathrm{~s}^{-1}$ (obtained from the FWHM of the broad He II component $\approx 1300 \mathrm{~km} \mathrm{~s}^{-1}$ ). The narrowness of the metal absorption lines associated with the PDLA suggests that only one or a few of the clumps within this outflowing material is present along our line of sight. Because the broad He II emission is spatially unresolved in our observations, this possible outflow should be confined on either nuclear or galactic scales. A schematic cartoon of the environment of QSO J0952+0114 is presented in Figure $10 .^{12}$

\footnotetext{
12 This schematic view is not representative of the spatial physical scales, because we know that the Ly $\alpha$ nebula has an extent of more than $100 \mathrm{pkpc}$ and the size of the PDLA is not well constrained, therefore the goal of this cartoon is to give a closer view of the QSO and PDLA zone.
}

Such clumpy outflow would naturally explain all the features observed in both emission and absorption on both small and large scales but is of course not unique, and in particular this scenario is degenerate with the possibility that the PDLA is intervening and that the peculiar broad He II emission is coming from the quasar BLR. In order to better disentangle this latter hypothesis, a statistical sample of quasars with similar spectra (see, e.g., Lusso et al. 2015; Jensen et al. 2016) observed with MUSE could be used in future studies to verify the presence of other "peculiar" broad He II emission and confirm, at least in a statistical sense, the scenario suggested above.

\subsection{The Origin of the Central Lya Emission}

Differently from the nebulae published in Borisova et al. (2016), we have the unique capability in the case of J0952 +0114 of tracing the nebular emission to innermost QSO regions because the PDLA acts as a coronagraph (as shown in Section 3, we also caution about the fact that our data are seeing-limited at the FWHM $\approx 0$ !" 7 level of the PSF). Therefore, if the central, narrow, and bright Ly $\alpha$ emission observed along our line of sight is not produced in the quasar BLR nor from the PDLA, what then is its origin? As we have argued in the previous section, the optically thick PDLA is only covering a small fraction of the ionizing opening angle of the quasar. As in the case of the illuminated CGM, the central component could indicate fluorescently emitting gas associated with: (a) the interstellar medium (ISM) of the quasar host galaxy, (b) centrally concentrated CGM material, (c) some of the less dense material of the hypothesized outflow containing the PDLA. A close inspection of the velocity maps of both Ly $\alpha$ and narrow He II emission in the central region as shown in Figure 4 suggests that the latter possibility could readily be excluded because we do not see any change in velocities consistent with the PDLA blueshift in the central regions. However, we do see clearly detected extended C IV emission, possibly suggesting that the inner regions have been enriched and possibly affected by (previous) outflow events. Interestingly, such extended C IV emission suggests that the J0952 +0114 nebula could be more metal-enriched than other MUSE QSO nebulae without PDLAs (B16, A19), and could possibly provide a link between the outflow observed in absorption and the metal emission properties. In order to establish a possible correlation, it is necessary to build a larger sample of quasars with PDLA and integral-field observations as already discussed in Finley et al. (2013) and Fathivavsari et al. (2018). Regarding the possibilities (a) and (b), we notice that both the SB profiles and velocity maps in the central regions do not show any abrupt changes (see Figures 3 and 4). These results suggest that the transition between CGM and ISM properties that determine the Ly $\alpha$ SB (e.g., density distribution and temperature) could be rather smooth or indistinguishable, at least in the region illuminated by the quasar. This is consistent with the recent results on the Slug Nebula $(\mathrm{C} 19)$ that have suggested that the density distribution of illuminated "cold" gas on CGM scales could be as broad as what is typically observed in the ISM.

\subsection{J0952+0114 and Other AGN Outflows}

QSO J0952+01144 is not the only case among observed high-redshift quasars for which possible signatures of outflows have been reported in the recent literature (e.g., Brusa et al. 2015; Bischetti et al. 2017; Fiore et al. 2017, among others). 
Despite the large number of studies, however, little is known about the detailed properties of the various phases that could be associated with these AGN outflows. In this respect, it could be interesting to consider some analogies between the possible clumpy J0952+0114 outflow and the molecular outflows published in the recent works of Cicone et al. (2015), Feruglio et al. (2017), and Bischetti et al. (2018). By using ALMA data (specifically the $\mathrm{CO}$ and $\mathrm{C}$ II emission), these authors found evidence of molecular outflows with a wide range of spatial extents (i.e., from a few kiloparsecs up to $30 \mathrm{kpc}$ ) in the halo of QSOs at redshifts $4<z<6$. In particular, a large fraction of the cold gas surrounding the quasars has been detected at higher velocities than the systemic ones (average excess of $1000 \mathrm{~km} \mathrm{~s}^{-1}$ ) and has been associated with possibly starforming regions. Their most plausible interpretation of the outflow properties, including mass, momentum rate, mass-loss rate, and kinetic power, is compatible with the predictions of the AGN-driven outflow models. Unfortunately, to date, we have no information on possible $\mathrm{CO}$ and $\mathrm{C}$ II extended emission from J0952+01144. Taken at face value, however, the velocities and spatial extent of molecular outflows reported above are similar to the scenario presented in this discussion, which suggests that the possible outflow associated with the PDLA could be consistent with other molecular outflows detected with ALMA.

\subsection{J0952+0114 QSO: Exotic or Ordinary Object?}

If the scenario presented above is correct, then the QSO $\mathrm{J} 0952+0114$ is likely not a truly "exotic" or extraordinary object but a QSO with similar intrinsic properties and environment to other luminous QSOs at this redshift, but with the serendipitous presence of a PDLA absorber along our line of sight. Indeed, the giant Ly $\alpha$ nebula that we have discovered around $\mathrm{J} 0952+0114$ is very similar to those observed by B16 and A19, which targeted typical QSOs without PDLAs. Other clumpy outflows can be present in other sources, and the rarity of such findings could reflect the small covering factor of such outflows rather than the peculiarity of J0952+0114, though dedicated follow-up observations, similar to those presented here, would be needed to confirm it with certainty.

\section{Summary and Conclusions}

In this paper, we presented a detailed study of $1 \mathrm{hr}$ MUSE integral-field spectroscopic observations of the radio-quiet QSO J0952 +0114 at $z=3.02$ with the goal of understanding the origin, location, and spatial extent of the gas associated with the PDLA. Our main findings can be summarized as follows.

1. We found a giant $(\approx 100 \mathrm{pkpc})$ Ly $\alpha$ nebula at $z \sim 3.017$ around $\mathrm{J} 0952+0114$. The Ly $\alpha$ properties of the gas, i.e., size, luminosity, and SB profile, enshrouding this QSO are not different from those of other MUSE QSO giant nebulae without PDLAs.

2. We also detected the presence of narrow and extended $\mathrm{C}$ IV and He II emission from the nebula at the same redshift as Ly $\alpha$.

3. In addition, we detected a bright spatially unresolved and relatively broad $\mathrm{He}$ II emission in the central region at the redshift of the PDLA, which possibly suggests the presence of a clumpy outflow on small scales that contains the PDLA.
4. We investigated the origin of the central regions of the nebula by analyzing the SB and velocity maps. The absence of abrupt changes in both properties suggests that we are witnessing a smooth transition between the CGM and the ISM in the region illuminated by the QSO.

5. Moreover, we performed a kinematical analysis based on both the velocity and velocity dispersion maps of the narrow Ly $\alpha, \mathrm{C}$ IV, and He II emission lines. The relatively low measured $v$ and $\sigma$ values do not strongly support the idea of an ongoing outflow on large scales.

6. The analysis of the maps of 2D He II/Ly $\alpha$ and C IV /Ly $\alpha$ line ratios revealed a positive gradient with increasing distance from the QSO, suggesting a non-homogeneous distribution of the ionization parameter of the CGM.

Thanks to the MUSE's performance, we have shown that the unusual QSO J0952+0114 has the same extended Ly $\alpha$ nebula as essentially all the radio-quiet QSOs at this redshift. Because of the fortuitous presence of the PDLA, this particular system enables us to shed more light on the physical properties of the relatively cold gas around J0952+0114 with unprecedented detail.

We thank Lutz Wisotzki for stimulating discussions. This work is based on observations taken at ESO/VLT in Paranal and we would like to thank the ESO staff for their assistance and support during the MUSE GTO campaigns. This work was supported by the Swiss National Science Foundation. This research made use of Astropy, a community-developed core PYTHON package for astronomy (Astropy Collaboration et al. 2013), NumPy and SciPy (Oliphant 2007), Matplotlib (Hunter 2007), IPython (Perez \& Granger 2007), and of the NASA Astrophysics Data System Bibliographic Services. S.C. and G.P. gratefully acknowledge support from Swiss National Science Foundation grant PP00P2_163824. A.F. acknowledges support from the ERC via Advanced Grant under grants agreement no. 339659-MUSICOS. J.B. acknowledges support by FCT/MCTES through national funds by grant UID/FIS/ 04434/2019 and through Investigador FCT Contract No. IF/ 01654/2014/CP1215/CT0003. S.D.J. is supported by a NASA Hubble Fellowship (HST-HF2-51375.001-A). T.N. acknowledges the Nederlandse Organisatie voor Wetenschappelijk Onderzoek (NWO) top grant TOP1.16.057.

\section{Appendix A \\ Identification of Possible Spectral Features at the Redshift of the PDLA}

In this appendix we show the results from the analysis of the J0952+0114 MUSE spectrum focused on the identification of possible emission and absorption features at the PDLA redshift. We performed the line identification in the continuumsubtracted spectrum of a $0 . " 6$ diameter region centered on the QSO. The only clear isolated emission line, located at the PDLA redshift, is the He II emission already presented in Figure 6. Figure 11 shows the other features detected at the PDLA redshift including a clear absorption system in the $\mathrm{C}$ IV wavelength region (middle panel) and $\mathrm{N} \mathrm{V}$ and $\mathrm{C} \mathrm{III]} \mathrm{emission}$ lines (left and right panels, respectively). 

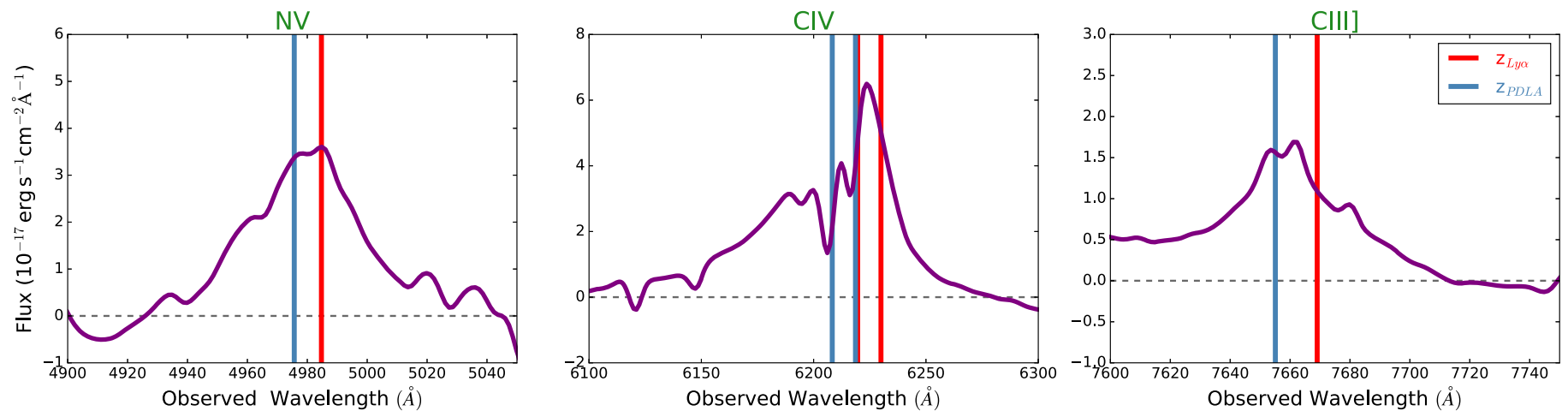

Figure 11. Expanded view of the $\mathrm{N} \mathrm{V}, \mathrm{C}$ IV, and C III] emission line regions. Blue vertical lines indicate the expected emission peaks corresponding to the PDLA redshift while the red ones correspond to the redshift obtained from the narrow Ly $\alpha$ emission.

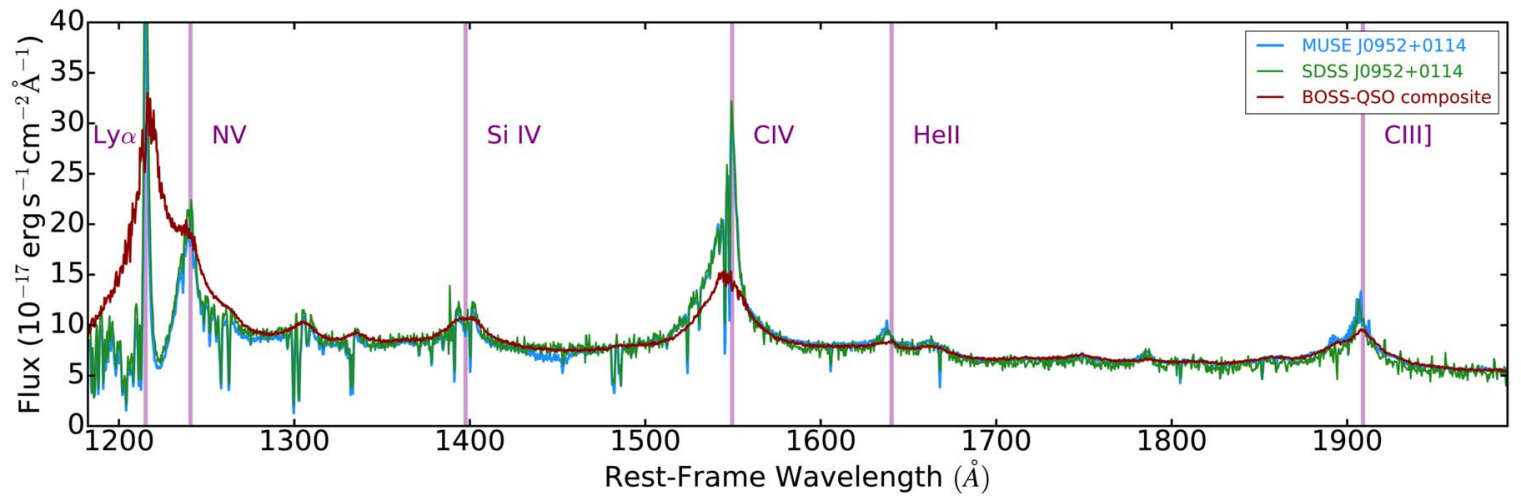

Figure 12. Comparison between the MUSE (blue), SDSS (green), and BOSS-QSO composite (dark red) spectra.

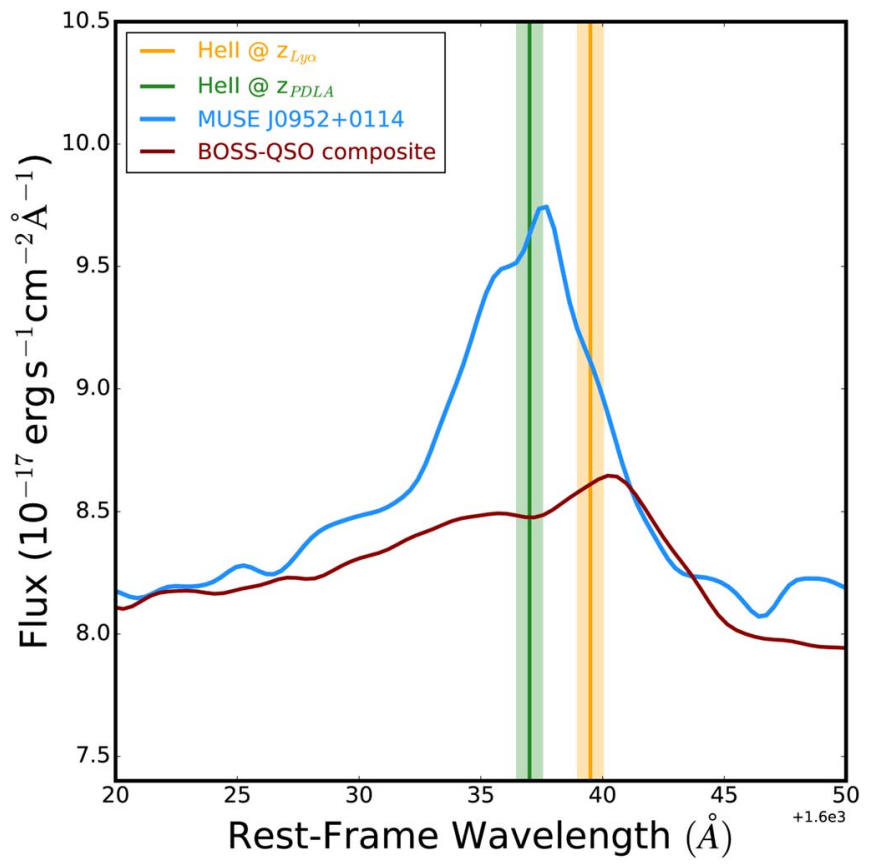

Figure 13. Zoom-in on the He II emission lines observed in the MUSE (blue) and the BOSS-QSO composite (dark red) spectra. The green and the orange vertical lines/areas indicate the position of the He II emission in the case of adopting the redshifts of the PDLA and the nebula, respectively.

\section{Appendix B \\ Comparison between the J0952+0114 MUSE Spectrum and Literature Data}

In this appendix, we provide a comparison between the J0952+0114 MUSE spectrum and literature data. In order to perform a consistent comparison with the SDSS spectrum (green solid line), we extracted our J0952+0114 spectrum (blue solid line) from a $3^{\prime \prime}$ diameter region. We note that the agreement between MUSE and SDSS is excellent. In addition, Figure 12 also includes the comparison with the BOSS-QSO composite spectrum of Jensen et al. (2016), which comprises 3106 QSO spectra with a median bolometric luminosity $\log \left(L_{\mathrm{bol}}\right)=46.66$, a median spectral index $\alpha=-1.52$, and a median redshift $z$ of 2.91. This particular composite spectrum has been chosen because it better matches the properties of $\mathrm{J} 0952+0114$ (i.e., $\log \left(L_{\mathrm{bol}}\right)=$ 46.48 and $z=3.02$ ). By comparing our J0952+0114 MUSE spectrum with the BOSS-QSO composite one, we found that the position and shape of the J0952+0114 He II emission line are significantly different from those of other QSOs with similar luminosity, redshift, and UV slope as highlighted in Figure 13.

\section{Appendix C \\ Comparison between the $\mathrm{J} 0952+0114$ and Literature $\mathrm{Ly} \alpha$ SB Profiles}

As shown in Figure 7, we have compared the circularly averaged SB profile of the J0952+0114 Ly $\alpha$ nebula with other Ly $\alpha$ SB profiles already published in the literature, i.e., B16 and A19. In the case of A19 we plotted an exponential profile (blue solid line) using the average values presented in their Table 4. In the case of the B16 MUSE Ly $\alpha$ SB profiles, we plotted the median profile (solid purple line) together with the 10th and 90th percentiles that are given in Table 2. 
Table 2

Average, Median, and Percentile Values of the MUSE Ly $\alpha$ SB Profiles Published in Borisova et al. (2016)

\begin{tabular}{|c|c|c|c|c|c|c|}
\hline $\begin{array}{l}\text { Radius } \\
(\mathrm{kpc})\end{array}$ & Average Ly $\alpha \mathrm{SB}^{\mathrm{a}}$ & Median Ly $\alpha$ SB & $\begin{array}{l}\text { 10th percentile } \\
\qquad\left(10^{-18} \mathrm{erg} \mathrm{s}^{-1}\right.\end{array}$ & $\begin{array}{l}\text { 25th percentile } \\
\left.\operatorname{arcsec}^{-2}\right)\end{array}$ & 75th percentile & 90th percentile \\
\hline 8.7 & 58.38 & 35.24 & 14.05 & 28.76 & 63.08 & 143.06 \\
\hline 10.9 & 45.78 & 30.92 & 8.19 & 15.76 & 44.78 & 116.99 \\
\hline 13.7 & 32.45 & 25.79 & 6.17 & 9.18 & 28.58 & 82.33 \\
\hline 17.3 & 23.80 & 15.07 & 4.81 & 5.50 & 18.22 & 57.84 \\
\hline 21.7 & 16.79 & 10.59 & 4.44 & 6.01 & 13.26 & 37.20 \\
\hline 27.4 & 11.80 & 6.67 & 2.02 & 3.83 & 10.73 & 29.00 \\
\hline 34.5 & 7.88 & 5.89 & 1.37 & 3.35 & 6.92 & 17.59 \\
\hline 43.4 & 5.56 & 4.29 & 1.06 & 2.39 & 6.10 & 11.30 \\
\hline 54.6 & 2.23 & 1.89 & 0.42 & 1.41 & 2.68 & 3.49 \\
\hline 68.8 & 0.97 & 0.64 & $0.05^{\mathrm{b}}$ & 0.27 & 1.52 & 2.00 \\
\hline 86.6 & 0.30 & 0.23 & $-0.48^{\mathrm{b}}$ & $-0.11^{\mathrm{b}}$ & 0.41 & 1.25 \\
\hline 109.0 & 0.07 & 0.10 & $-0.71^{b}$ & $-0.05^{b}$ & 0.21 & 0.55 \\
\hline
\end{tabular}

Notes. All SB values were rescaled to $z=3$.

${ }^{\mathrm{a}}$ The average values presented here are computed on a linear scale. Note that in B16 they were computed in a logarithmic scale.

b These values are not significant because they are affected by noise.

\section{ORCID iDs}

Raffaella Anna Marino (iD https://orcid.org/0000-00028559-6565

Simon J. Lilly (i) https://orcid.org/0000-0002-6423-3597

Jorryt Matthee (i) https://orcid.org/0000-0003-2871-127X

Jarle Brinchmann (1) https://orcid.org/0000-0003-4359-8797

Sowgat Muzahid (iD https://orcid.org/0000-0003-3938-8762

Ilane Schroetter (iD https://orcid.org/0000-0002-1099-7401

Themiya Nanayakkara (i) https://orcid.org/0000-00032804-0648

\section{References}

Arrigoni Battaia, F., Hennawi, J. F., Prochaska, J. X., et al. 2019, MNRAS, 482, 3162

Arrigoni Battaia, F., Yang, Y., Hennawi, J. F., et al. 2015, ApJ, 804, 26

Astropy Collaboration, Robitaille, T. P., Tollerud, E. J., et al. 2013, A\&A, 558, A33

Bacon, R., Accardo, M., Adjali, L., et al. 2010, Proc. SPIE, 7735, 773508

Bischetti, M., Maiolino, R., Fiore, S., et al. 2018, arXiv:1806.00786

Bischetti, M., Piconcelli, E., Vietri, G., et al. 2017, A\&A, 598, 122

Borisova, E., Cantalupo, S., Lilly, S. J., et al. 2016, ApJ, 831, 39

Brusa, M., Bongiorno, A., Cresci, G., et al. 2015, MNRAS, 446, 2394

Cantalupo, S. 2017, in Gas Accretion onto Galaxies, Astrophysics and Space Science Library, Vol. 430, ed. A. Fox \& R. Davé (Cham: Springer), 195

Cantalupo, S., Pezzulli, G., Lilly, S. J., et al. 2019, MNRAS, 483, 5188

Cicone, C., Maiolino, R., Gallerani, S., et al. 2015, A\&A, 574, 14

Corlies, L., Peeples, M. S., Tumlinson, J., et al. 2018, arXiv:1811.05060

Dawson, K. S., Schlegel, D. J., Ahn, C. P., et al. 2013, AJ, 145, 10
Dijkstra, M., Haiman, Z., \& Spaans, M. 2006, ApJ, 649, 14

Fathivavsari, H., Petitjean, P., Jamialahmadi, N., et al. 2018, MNRAS, 477, 5625

Fathivavsari, H., Petitjean, P., Noterdaeme, P., et al. 2016, MNRAS, 461, 1816

Feltre, A., Charlot, S., \& Gutkin, J. 2016, MNRAS, 456, 3354

Feruglio, C., Ferrara, A., Bischetti, M., et al. 2017, A\&A, 608, 30

Finley, H., Petitjean, P., Pâris, I., et al. 2013, A\&A, 558, A111

Fiore, F., Feruglio, C., Shankar, F., et al. 2017, A\&A, 601, A143

Ginolfi, M., Maiolino, R., Carniani, S., et al. 2018, MNRAS, 476, 2421

Hall, P. B., Snedden, S. A., Niederste-Ostholt, M., et al. 2004, AJ, 128, 534

Hinshaw, G., Larson, D., Komatsu, E., et al. 2013, ApJS, 208, 19

Hunter, J. D. 2007, CSE, 9, 90

Ivezić, Ž., Menou, K., Knapp, G. R., et al. 2002, AJ, 124, 2364

Jensen, T. W., Vivek, M., Dawson, K. S., et al. 2016, ApJ, 833, 199

Jiang, P., Zhou, H., Pan, X., et al. 2016, ApJ, 821, 1

Leibler, C. N., Cantalupo, S., Holden, B. P., \& Madau, P. 2018, MNRAS, 480, 2094

Lira, P., Kaspi, S., Netzer, H., et al. 2018, ApJ, 865, 56

Lusso, E., Worseck, G., Hennawi, J. F., et al. 2015, MNRAS, 449, 4204

Marino, R. A., Cantalupo, S., Lilly, S. J., et al. 2018, ApJ, 859, 53

McCourt, M., Oh, S. P., O'Leary, R., \& Madigan, A. 2018, MNRAS, 473, 5407

Neufeld, D. A. 1991, ApJL, 370, L85

North, P. L., Marino, R. A., Gorgoni, C., et al. 2017, A\&A, 604, 23

Oke, J. B., \& Gunn, J. E. 1983, ApJ, 266, 713

Oliphant, T. E. 2007, CSE, 9, 10

Perez, F., \& Granger, B. E. 2007, CSE, 9, 21

Prescott, M. K. M., Martin, C. L., \& Dey, A. 2015, ApJ, 799, 62

Schneider, D. P., Fan, X., Hall, P. B., et al. 2003, AJ, 126, 2579

Villar-Martin, M., Tadhunter, C., \& Clark, N. 1997, A\&A, 323, 21

Weilbacher, P. M. 2015, Science Operations 2015: Science Data Management, Zenodo, doi:10.5281/zenodo.3465

York, D. G., Adelman, J., Anderson, J. E., Jr., et al. 2000, AJ, 120, 1579 